\title{
ON THE LOCAL WELL-POSEDNESS OF THE BENJAMIN-ONO AND MODIFIED BENJAMIN-ONO EQUATIONS
}

\author{
Carlos E. Kenig and Kenneth D. Koenig
}

Abstract. We prove that the Benjamin-Ono equation is locally well-posed in $H^{s}(\mathbf{R})$ for $s>9 / 8$ and that for arbitrary initial data, the modified (cubic nonlinearity) Benjamin-Ono equation is locally well-posed in $H^{s}(\mathbf{R})$ for $s \geq 1$.

\section{Introduction}

We consider the initial value problems for the Benjamin-Ono (BO) equation

$$
\left\{\begin{array}{l}
\partial_{t} u+H \partial_{x}^{2} u+u \partial_{x} u=0 \quad(x, t) \in \mathbf{R}^{2} \\
u(x, 0)=u_{0}(x)
\end{array}\right.
$$

and a modified Benjamin-Ono (mBO) equation (with cubic nonlinearity)

$$
\left\{\begin{array}{l}
\partial_{t} u+H \partial_{x}^{2} u+u^{2} \partial_{x} u=0 \quad(x, t) \in \mathbf{R}^{2} \\
u(x, 0)=u_{0}(x)
\end{array}\right.
$$

where $H$ is the Hilbert transform.

The Benjamin-Ono equation models behavior of long internal waves in deep stratified fluids $([\mathrm{B}],[\mathrm{O}])$. Both equations satisfy the conservation laws

$$
\begin{aligned}
& I_{1}(u)=\int_{-\infty}^{\infty} u(x, t) d x \\
& I_{2}(u)=\int_{-\infty}^{\infty} u^{2}(x, t) d x
\end{aligned}
$$

and the Benjamin-Ono equation possesses an infinite number of conservation laws, including

$$
\begin{aligned}
& I_{3}(u)=\int_{-\infty}^{\infty}\left(\left|D_{x}^{\frac{1}{2}} u(x, t)\right|^{2}+\frac{1}{3} u^{3}(x, t)\right) d x \\
& I_{4}(u)=\int_{-\infty}^{\infty}\left(\left(\partial_{x} u\right)^{2}(x, t)+\frac{3}{4}\left(u^{2} D_{x} u\right)(x, t)+\frac{1}{8} u^{4}(x, t)\right) d x .
\end{aligned}
$$

The Benjamin-Ono equation has global weak solutions in $L^{2}(\mathbf{R}), H^{\frac{1}{2}}(\mathbf{R})$, and $H^{1}(\mathbf{R})([\mathrm{GV}],[\mathrm{To}],[\mathrm{Sa}])$, and it has been known for some time that it is globally

Received August 14, 2003. 
well-posed in $H^{s}(\mathbf{R})$ for $s \geq 3 / 2$ (see $[\mathrm{P}]$ and the references therein). Recently, H. Koch and N. Tzvetkov [KoTz] proved local well-posedness for $s>5 / 4$ by a substantially simpler argument. Our first result is the following improvement:

Theorem 1.1. Let $s>9 / 8$. For any $u_{0} \in H^{s}(\mathbf{R})$, there exists $T \gtrsim\left\|u_{0}\right\|_{H^{s}}^{-4}$ and a unique solution $u$ of the Benjamin-Ono equation (1.1) satisfying

$$
u \in C\left([0, T]: H^{s}(\mathbf{R})\right) \quad \text { and } \quad \partial_{x} u \in L^{1}\left([0, T]: L^{\infty}(\mathbf{R})\right) .
$$

Moreover, for any $R>0$, the map $u_{0} \mapsto u(t)$ is continuous from the ball $\left\{u_{0} \in\right.$ $\left.H^{s}(\mathbf{R}):\left\|u_{0}\right\|_{H^{s}}<R\right\}$ to $C\left([0, T]: H^{s}(\mathbf{R})\right)$.

Observe that a desirable goal is to extend local well-posedness to $s \geq 1$, since global well-posedness would then hold in $H^{1}(\mathbf{R})$ due to the conservation law (1.4). Just as this paper was completed, T. Tao ([Ta]) announced a proof of this global well-posedness in $H^{1}(\mathbf{R})$, by performing an appropriate gauge transformation that eliminates the derivative (on high-frequency components) in the nonlinear term. Nevertheless, we expect that the simplicity of our argument for the range $s>9 / 8$, and its wide scope of applicability, should be of independent interest. For instance, a very similar proof to the one given here yields, for the "dispersion-generalized" Benjamin-Ono equation

$$
\left\{\begin{array}{l}
\partial_{t} u+\partial_{x} D_{x}^{1+a} u+u \partial_{x} u=0 \quad(x, t) \in \mathbf{R}^{2}, \quad 0 \leq a<1 \\
u(x, 0)=u_{0}(x)
\end{array}\right.
$$

local well-posedness in $H^{s}(\mathbf{R})$ for $s>\frac{9}{8}-\frac{3 a}{8}$. This improves the best previously known result given in [KPV1], where local well-posedness was proved for $s>$ $\frac{3}{2}-\frac{3 a}{4}$. Since these equations are not completely integrable (for $0<a<1$ ), it is unclear whether Tao's gauge transformation applies to them.

For the modified Benjamin-Ono equation (1.2), it has been known that it is locally well-posed for $s>3 / 2$ ([I]) and, for small initial data, for $s>1$ ([KPV3]). Also very recently, the latter result (i.e. for small data) was extended to $s>1 / 2$ by L. Molinet and F. Ribaud ([MR]). We show that for arbitrary initial data, $\mathrm{mBO}$ is locally well-posed in $H^{s}(\mathbf{R})$ for $s \geq 1$.

Theorem 1.2. Let $s \geq 1$. For any $u_{0} \in H^{s}(\mathbf{R})$, there exists $T \gtrsim \min \left(1,\left\|u_{0}\right\|_{H^{1}}^{-25}\right)$ and a unique solution $u$ of the modified Benjamin-Ono equation (1.2) satisfying

$$
u \in C\left([0, T]: H^{s}(\mathbf{R})\right) \quad \text { and } \quad \partial_{x} u \in L^{4}\left([0, T]: L^{\infty}(\mathbf{R})\right) .
$$

Moreover, for any $R>0$, the map $u_{0} \mapsto u(t)$ is continuous from the ball $\left\{u_{0} \in\right.$ $\left.H^{s}(\mathbf{R}):\left\|u_{0}\right\|_{H^{s}}<R\right\}$ to $C\left([0, T]: H^{s}(\mathbf{R})\right)$.

Our method is to refine the energy method and smoothing effect approach (such as in [KPV1], taking advantage of the Christ-Kiselev lemma 2.5 below). It is worth pointing out that it is not possible to use the contraction principle to prove local well-posedness in $H^{s}(\mathbf{R})$ for the Benjamin-Ono equation ([MSaTz]). On the other hand, the results of Kenig-Ponce-Vega and Molinet-Ribaud cited above for modified Benjamin-Ono were proved by contraction methods. These cannot apply for $s<1 / 2$, since $\mathrm{mBO}$ is not $C^{3}$ well-posed in this range ([MR]). 
The following notation will be used throughout this article: $D^{s}=(-\Delta)^{s / 2}$ and $J^{s}=(I-\Delta)^{s / 2}$ denote the Riesz and Bessel potentials of order $-s$, respectively. We write

$$
\begin{aligned}
\|f\|_{L_{x}^{p} L_{t}^{q}} & =\|\| f(x, \cdot)\left\|_{L^{q}(\mathbf{R})}\right\|_{L^{p}(\mathbf{R})} \\
\|f\|_{L_{x}^{p} L_{T}^{q}} & =\|\| f(x, \cdot)\left\|_{L^{q}([0, T])}\right\|_{L^{p}(\mathbf{R})}
\end{aligned}
$$

with similar definitions for $L_{t}^{q} L_{x}^{p}$ and $L_{T}^{q} L_{x}^{p}$. Finally, we say $A \lesssim B$ if there exists a constant $c>0$ such that $A \leq c B$ (it will be clear from context what parameters $c$ may depend on).

\section{Linear estimates and local smoothing}

In this section, we provide the linear estimates and local smoothing properties for solutions to $\mathrm{BO}$ and $\mathrm{mBO}$. Consider the corresponding linear IVP

$$
\left\{\begin{array}{l}
\partial_{t} v+H \partial_{x}^{2} v=0 \quad(x, t) \in \mathbf{R}^{2} \\
v(x, 0)=v_{0}(x)
\end{array}\right.
$$

and

$$
\left\{\begin{array}{l}
\partial_{t} w+H \partial_{x}^{2} w=f \quad(x, t) \in \mathbf{R}^{2} \\
w(x, 0)=0
\end{array}\right.
$$

whose solutions are given by

$$
\begin{aligned}
v(x, t) & =S(t) v_{0}(x) \\
w(x, t) & =\int_{0}^{t} S\left(t-t^{\prime}\right) f\left(\cdot, t^{\prime}\right) d t^{\prime}
\end{aligned}
$$

where $S(t) u_{0}(x)=c \int_{-\infty}^{\infty} e^{i x \xi} e^{i t \xi|\xi|} \hat{u}_{0}(\xi) d \xi$.

We first state the standard Strichartz estimate and sharp Kato smoothing effect for the unitary group $\{S(t)\}_{t \in \mathbf{R}}$ (see e.g. [KPV1]).

Lemma 2.1. For $v_{0} \in L^{2}$,

$$
\left\|S(t) v_{0}\right\|_{L_{t}^{q} L_{x}^{p}} \leq c\left\|v_{0}\right\|_{L^{2}} \quad \text { with }(q, p)=\left(\frac{4}{\theta}, \frac{2}{1-\theta}\right)
$$

for any $\theta \in[0,1]$, and

$$
\left\|D_{x}^{1 / 2} S(t) v_{0}\right\|_{L_{x}^{\infty} L_{t}^{2}}=c\left\|v_{0}\right\|_{L^{2}}
$$

The following version of the local smoothing effect for solutions to BO was established in [KPV1]; a careful examination of the proof ${ }^{1}$ shows that the result extends (as stated below) to solutions of $\mathrm{mBO}$ as well.

\footnotetext{
${ }^{1}$ Here it is necessary to use the energy estimate for mBO given in Lemma 2.7.
} 
Lemma 2.2. Fix $\delta>0$, and let $I$ be an interval of unit length. If $u \in C([0, T]$ : $\left.H^{s+1}(\mathbf{R})\right)$ is a solution to $B O$, then

$$
\begin{array}{r}
\left(\int_{0}^{T} \int_{I}\left|D_{x}^{s-\frac{1}{2}} \partial_{x} u(x, t)\right|^{2} d x d t\right)^{1 / 2} \leq c\left\|u_{0}\right\|_{H^{s}}\left(1+T+T\left\|u_{0}\right\|_{H^{\frac{1}{2}+\delta}}+\left\|\partial_{x} u\right\|_{L_{T}^{1} L_{x}^{\infty}}\right) \\
\cdot \exp \left(c .5 \partial_{x} u \|_{L_{T}^{1} L_{x}^{\infty}}\right)
\end{array}
$$

for $s \geq \frac{1}{2}$. Similarly, if $u$ is a solution to $m B O$, then

$$
\begin{array}{r}
\left(\int_{0}^{T} \int_{I}\left|D_{x}^{s-\frac{1}{2}} \partial_{x} u(x, t)\right|^{2} d x d t\right)^{1 / 2} \leq c\left\|u_{0}\right\|_{H^{s}}\left(1+T+(1+T)\left\|u_{0}\right\|_{H^{\frac{1}{2}+\delta}}+\left\|\partial_{x} u\right\|_{L_{T}^{1} L_{x}^{\infty}}\right) \\
\quad \cdot \exp \left(c\|u\|_{L_{T}^{2} L_{x}^{\infty}}^{2}\right) \exp \left(c\left\|\partial_{x} u\right\|_{L_{T}^{2} L_{x}^{\infty}}^{2}\right) .
\end{array}
$$

Next we recall the maximal function estimate proved in [KPV1].

Lemma 2.3. Assume $v_{0} \in H^{\frac{1}{2}+\delta}$ for some fixed $\delta>0$. Then

$$
\left\|S(t) v_{0}\right\|_{L_{x}^{2} L_{T}^{\infty}} \leq\left(\sum_{j=-\infty}^{\infty}\left\|S(t) v_{0}\right\|_{L^{\infty}([j, j+1) \times[0, T])}^{2}\right)^{1 / 2} \leq c(1+T)\left\|v_{0}\right\|_{H^{\frac{1}{2}+\delta}} .
$$

Using duality arguments and complex interpolation, we combine the Katotype smoothing and maximal function estimates to obtain additional linear estimates needed in the proof of Theorem 1.2.

\section{Lemma 2.4.}

(a) For $T \in[0,1]$,

$$
\left\|\partial_{x} \int_{0}^{t} S\left(t-t^{\prime}\right) f\left(\cdot, t^{\prime}\right) d t^{\prime}\right\|_{L_{T}^{4} L_{x}^{\infty}} \leq c\left\|D_{x}^{1 / 2} f\right\|_{L_{x}^{1} L_{T}^{2}} .
$$

(b) For $\delta, \theta, T \in[0,1]$ and $\varepsilon>0$,

$$
\left\|D_{x}^{\theta} S(t) v_{0}\right\|_{L_{x}^{2 /(1-\theta)} L_{T}^{2 / \theta}} \leq c\left\|D_{x}^{\frac{1}{2}+\varepsilon} v_{0}\right\|_{L^{2}}+c\left\|v_{0}\right\|_{L^{2}}
$$

and

$\left\|D_{x}^{\theta} \int_{0}^{t} S\left(t-t^{\prime}\right) f\left(\cdot, t^{\prime}\right) d t^{\prime}\right\|_{L_{x}^{2 /(1-\theta)} L_{T}^{2 / \theta}} \leq c T^{\frac{\delta(1-\theta)}{2}}\left\|D_{x}^{\frac{\delta(1-\theta)}{2}+\varepsilon} f\right\|_{L_{x}^{p} L_{T}^{2}}+c T^{1 / 2}\|f\|_{L_{x}^{2} L_{T}^{2}}$

where $p=p(\delta, \theta)=\frac{2}{2-\delta(1-\theta)}$.

A useful lemma of Christ and Kiselev ([ChKi]) allows one to deduce the inequalities (2.8) and (2.10) from the corresponding "nonretarded" ones. The version of this lemma that we use is the one presented and proved in $[\mathrm{MR}]$, [SmSo]. 
Lemma 2.5. Let $\mathcal{T} f(t)=\int_{-\infty}^{\infty} K\left(t, t^{\prime}\right) f\left(t^{\prime}\right) d t^{\prime}$ be a linear operator, where $K$ : $\mathcal{S}\left(\mathbf{R}^{2}\right) \rightarrow C\left(\mathbf{R}^{3}\right)$. Assume that $\|\mathcal{T} f\|_{L_{x}^{p_{1}} L_{t}^{q_{1}}} \leq c\|f\|_{L_{x}^{p_{2} L_{t}^{q_{2}}}}$ for some $p_{1}, p_{2}, q_{1}, q_{2} \in$ $[1, \infty]$ with

$$
\min \left(p_{1}, q_{1}\right)>\max \left(p_{2}, q_{2}\right)
$$

Then

$$
\left\|\int_{0}^{t} K\left(t, t^{\prime}\right) f\left(t^{\prime}\right) d t^{\prime}\right\|_{L_{x}^{p_{1}} L_{t}^{q_{1}}} \leq c\|f\|_{L_{x}^{p_{2}} L_{t}^{q_{2}} .}
$$

A similar result ${ }^{2}$ holds for the spaces $L_{t}^{q_{1}} L_{x}^{p_{1}}$ and/or $L_{t}^{q_{2}} L_{x}^{p_{2}}$ instead of $L_{x}^{p_{1}} L_{t}^{q_{1}}$, $L_{x}^{p_{2}} L_{t}^{q_{2}}$. Moreover, the case $q_{1}=\infty ; p_{2}, q_{2}<\infty$ is allowed even if (2.11) fails; more precisely, if $T \leq 1$ and $\|\mathcal{T} f\|_{L_{x}^{p_{1}} L_{T}^{\infty}} \leq c\|f\|_{L_{x}^{p_{2}} L_{T}^{q_{2}}}$ with $p_{2} \neq \infty, q_{2} \neq \infty$, then

$$
\left\|\int_{0}^{t} K\left(t, t^{\prime}\right) f\left(t^{\prime}\right) d t^{\prime}\right\|_{L_{x}^{p_{1}} L_{T}^{\infty}} \leq c\|f\|_{L_{x}^{p_{2}} L_{T}^{q_{2}}}
$$

Proof of Lemma 2.4.

(a) A standard $T T^{*}$ argument using Lemma 2.1 yields the linear estimate

$$
\left\|\partial_{x} \int_{-\infty}^{\infty} S\left(t-t^{\prime}\right) f\left(\cdot, t^{\prime}\right) d t^{\prime}\right\|_{L_{t}^{4} L_{x}^{\infty}} \leq c\left\|D_{x}^{1 / 2} f\right\|_{L_{x}^{1} L_{t}^{2}} .
$$

Indeed, the $L_{t}^{4} L_{x}^{\infty}$ Strichartz estimate and smoothing effect (2.4) imply that

$$
\begin{aligned}
& \left\|S(t) \int_{-\infty}^{\infty} H D_{x}^{1 / 2} S\left(-t^{\prime}\right)\left(D_{x}^{1 / 2} f\right)\left(t^{\prime}\right) d t^{\prime}\right\|_{L_{t}^{4} L_{x}^{\infty}} \\
\lesssim & \left\|\int_{-\infty}^{\infty} D_{x}^{1 / 2} S\left(-t^{\prime}\right)\left(D_{x}^{1 / 2} f\right)\left(t^{\prime}\right) d t^{\prime}\right\|_{L_{x}^{2}} \\
\lesssim & \sup _{\|g\|_{L^{2}} \leq 1}\left|\int_{-\infty}^{\infty} \int_{-\infty}^{\infty} D_{x}^{1 / 2} S\left(-t^{\prime}\right)\left(D_{x}^{1 / 2} f\right)\left(x, t^{\prime}\right) g(x) d t^{\prime} d x\right| \\
\lesssim \quad & \sup _{\|g\|_{L^{2}} \leq 1}\left|\int_{-\infty}^{\infty} \int_{-\infty}^{\infty} D_{x}^{1 / 2} f\left(x, t^{\prime}\right) D_{x}^{1 / 2} S\left(t^{\prime}\right) g(x) d x d t^{\prime}\right| \\
\lesssim \quad & \sup _{\|g\|_{L^{2}} \leq 1}\left\|D_{x}^{1 / 2} f\right\|_{L_{x}^{1} L_{t}^{2}}\left\|D_{x}^{1 / 2} S(t) g\right\|_{L_{x}^{\infty} L_{t}^{2}} \leq\left\|D_{x}^{1 / 2} f\right\|_{L_{x}^{1} L_{t}^{2} .}
\end{aligned}
$$

Therefore, by Lemma 2.5,

$$
\left\|\partial_{x} \int_{0}^{t} S\left(t-t^{\prime}\right) f\left(\cdot, t^{\prime}\right) d t^{\prime}\right\|_{L_{t}^{4} L_{x}^{\infty}} \leq c\left\|D_{x}^{1 / 2} f\right\|_{L_{x}^{1} L_{t}^{2}}
$$

which in turn implies (2.8).

\footnotetext{
${ }^{2}$ The $L_{t}^{q_{1}} L_{x}^{p_{1}}, L_{x}^{p_{2}} L_{t}^{q_{2}}$ (resp. $L_{x}^{p_{1}} L_{t}^{q_{1}}, L_{t}^{q_{2}} L_{x}^{p_{2}}$; or $\left.L_{t}^{q_{1}} L_{x}^{p_{1}}, L_{t}^{q_{2}} L_{x}^{p_{2}}\right)$ version of the ChristKiselev lemma holds with the condition $q_{1}>\max \left(p_{2}, q_{2}\right)\left(\right.$ resp. $\min \left(p_{1}, q_{1}\right)>q_{2}$; or $\left.q_{1}>q_{2}\right)$ instead of (2.11).
} 
(b) The proof is in part similar ${ }^{3}$ to the one in [KPV3] that showed

$$
\left\|D_{x}^{\frac{3 \theta-1}{4}} S(t) v_{0}\right\|_{L_{x}^{4 /(1-\theta)} L_{t}^{2 / \theta}} \leq c\left\|v_{0}\right\|_{L^{2}}
$$

and

$$
\left\|D_{x}^{\frac{5 \theta}{4}} \int_{0}^{t} S\left(t-t^{\prime}\right) f\left(\cdot, t^{\prime}\right) d t^{\prime}\right\|_{L_{x}^{4 /(1-\theta)} L_{t}^{2 / \theta}} \leq c T^{\frac{1-\theta}{2}}\left\|D_{x}^{1 / 4} f\right\|_{L_{x}^{2 /(1+\theta)} L_{T}^{2}} .
$$

To adapt the argument to the operator $D_{x}^{1 / 2}\left(\right.$ instead of $\left.D_{x}^{1 / 4}\right)$, one interpolates the smoothing effect

$$
\left\|D_{x}^{1+i \alpha} S(t) v_{0}\right\|_{L_{x}^{\infty} L_{t}^{2}} \leq c\left\|v_{0}\right\|_{H^{\frac{1}{2}}}
$$

with

$$
\left\|D_{x}^{i \alpha} S(t) v_{0}\right\|_{L_{x}^{2} L_{T}^{\infty}} \leq c\left\|v_{0}\right\|_{H^{\frac{1}{2}+\varepsilon}}
$$

to obtain (2.9). For (2.10), we decompose $f$ into small and large frequencies (using smooth cutoffs) so that $f=f_{1}+f_{2}$ with supp $\hat{f}_{1} \subset\{|\xi|<2\}$ and supp $\hat{f}_{2} \subset$ $\{|\xi|>1\}$. Recall that for any fixed $\kappa>0$, there exist finite measures $\mu, \nu$ such that $|\xi|^{\kappa}=\left(1+|\xi|^{2}\right)^{\kappa / 2} \hat{\mu}(\xi)$ and $\left(1+|\xi|^{2}\right)^{\kappa / 2}=\left(1+|\xi|^{\kappa}\right) \hat{\nu}(\xi)$ (see for example [St], pp. 133-134). Therefore, combining the inequalities $\left\|D_{x}^{\frac{1}{2}} J^{i \alpha} S(t) u_{0}\right\|_{L_{x}^{\infty} L_{T}^{2}} \leq$ $c\left\|u_{0}\right\|_{L^{2}}$ and $\left\|S(t) u_{0}\right\|_{L_{x}^{2} L_{T}^{\infty}} \leq c\left\|J^{\frac{1}{2}+\varepsilon} u_{0}\right\|_{L^{2}}$ yields (by a $T T^{*}$ argument as above)

$$
\left\|J_{x}^{i \alpha-\varepsilon} \int_{-\infty}^{\infty} S\left(t-t^{\prime}\right) f_{2}\left(\cdot, t^{\prime}\right) d t^{\prime}\right\|_{L_{x}^{2} L_{T}^{\infty}} \lesssim\left\|f_{2}\right\|_{L_{x}^{1} L_{T}^{2}}
$$

and hence

$$
\left\|J_{x}^{i \alpha-\varepsilon} \int_{0}^{t} S\left(t-t^{\prime}\right) f_{2}\left(\cdot, t^{\prime}\right) d t^{\prime}\right\|_{L_{x}^{2} L_{T}^{\infty}} \lesssim\left\|f_{2}\right\|_{L_{x}^{1} L_{T}^{2}}
$$

by Lemma 2.5. On the other hand, by Lemma 2.3 ,

$$
\left\|J_{x}^{-\frac{1}{2}-\varepsilon+i \alpha} \int_{0}^{t} S\left(t-t^{\prime}\right) f_{2}\left(\cdot, t^{\prime}\right) d t^{\prime}\right\|_{L_{x}^{2} L_{T}^{\infty}} \lesssim T^{1 / 2}\left\|f_{2}\right\|_{L_{x}^{2} L_{T}^{2}} .
$$

Interpolating (2.14) and (2.15) gives

$$
\left\|J_{x}^{-\frac{\delta}{2}-\varepsilon+i \alpha} \int_{0}^{t} S\left(t-t^{\prime}\right) f_{2}\left(\cdot, t^{\prime}\right) d t^{\prime}\right\|_{L_{x}^{2} L_{T}^{\infty}} \lesssim T^{\frac{\delta}{2}}\left\|f_{2}\right\|_{L_{x}^{2 /(2-\delta)} L_{T}^{2}}
$$

for $\delta \in[0,1]$.

\footnotetext{
${ }^{3}$ The direct analogue of $(2.13)$ is

$$
\left\|D_{x}^{\frac{3 \theta}{2}} \int_{0}^{t} S\left(t-t^{\prime}\right) f\left(\cdot, t^{\prime}\right) d t^{\prime}\right\|_{L_{x}^{2 /(1-\theta)} L_{T}^{2 / \theta}} \leq c T^{\frac{1-\theta}{2}}\left\|D_{x}^{\frac{1}{2}+\varepsilon} f\right\|_{L_{x}^{2 /(1+\theta)} L_{T}^{2}}+c T^{1 / 2}\|f\|_{L_{x}^{2} L_{T}^{2}} .
$$
}

but the extra $\varepsilon$-derivative in the $\left\|D_{x}^{\frac{1}{2}+\varepsilon} f\right\|_{L_{x}^{2 /(1+\theta)} L_{T}^{2}}$ term causes difficulties when this estimate is applied with $f=u^{2} \partial_{x} u$ (see (4.2) below). 
Now we also know the following analogue of the smoothing effect (2.4):

$$
\left\|J_{x}^{1+i \alpha} \int_{0}^{t} S\left(t-t^{\prime}\right) f_{2}\left(\cdot, t^{\prime}\right) d t^{\prime}\right\|_{L_{x}^{\infty} L_{T}^{2}} \lesssim\left\|f_{2}\right\|_{L_{x}^{1} L_{T}^{2}}
$$

(see (2.9) in [KPV3], where $(2.17)$ is proved with $D_{x}^{1+i \alpha}$ instead of $J_{x}^{1+i \alpha}$ ), so interpolating (2.16) and (2.17) yields the desired result (2.10) for $f_{2}$ (with just the first term on the right-hand side). For $f_{1}$, it is easiest to estimate the lefthand side of (2.10) directly, using the result (2.9) already established. Thus

$$
\begin{aligned}
\left\|D_{x}^{\theta} \int_{0}^{t} S\left(t-t^{\prime}\right) f_{1}\left(\cdot, t^{\prime}\right) d t^{\prime}\right\|_{L_{x}^{2 /(1-\theta)} L_{T}^{2 / \theta}} & \leq \int_{0}^{T}\left\|D_{x}^{\theta} S(t) S\left(-t^{\prime}\right) f_{1}\left(\cdot, t^{\prime}\right)\right\|_{L_{x}^{2 /(1-\theta)} L_{T}^{2 / \theta}} d t^{\prime} \\
& \lesssim \int_{0}^{T}\left(\left\|D_{x}^{\frac{1}{2}+\varepsilon} f_{1}\right\|_{L_{x}^{2}}+\left\|f_{1}\right\|_{L_{x}^{2}}\right) d t \\
& \lesssim T^{1 / 2}\left\|f_{1}\right\|_{L_{x}^{2} L_{T}^{2}} \\
& \lesssim T^{1 / 2}\|f\|_{L_{x}^{2} L_{T}^{2}}
\end{aligned}
$$

Remark: By (2.7) and the proof of the last statement in Lemma 2.5, we obtain the following analogue of (2.16): for any $\delta<1$, there exists $p \in(1,2)$ such that

$$
\left\|\int_{0}^{t} S\left(t-t^{\prime}\right) f\left(\cdot, t^{\prime}\right) d t^{\prime}\right\|_{\ell_{j}^{2}\left(L^{\infty}([j, j+1) \times[0, T])\right)} \lesssim T^{\frac{\delta}{2}}\left\|D_{x}^{\frac{1}{2}} f\right\|_{L_{x}^{p} L_{T}^{2}}+T^{1 / 2}\|f\|_{L_{x}^{2} L_{T}^{2}} .
$$

To analyze the products that arise from the nonlinear term of the $\mathrm{BO}$ and $\mathrm{mBO}$ equations, we require the following Leibniz rules for fractional derivatives. For detailed proofs of these facts, see [KPV2].

\section{Lemma 2.6.}

(a) Let $\alpha=\alpha_{1}+\alpha_{2} \in(0,1)$ with $\alpha_{i} \in(0, \alpha), p \in[1, \infty)$, and $p_{1}, p_{2} \in(1, \infty)$ such that $\frac{1}{p}=\frac{1}{p_{1}}+\frac{1}{p_{2}}$. Then

$$
\left\|D^{\alpha}(f g)-f D^{\alpha} g-g D^{\alpha} f\right\|_{L^{p}} \leq c\left\|D^{\alpha_{1}} f\right\|_{L^{p_{1}}}\left\|D^{\alpha_{2}} g\right\|_{L^{p_{2}}} .
$$

Moreover, if $p>1$, then the case $\alpha_{2}=0,1<p_{2} \leq \infty$ is also allowed.

(b) Let $\alpha=\alpha_{1}+\alpha_{2} \in(0,1)$ with $\alpha_{i} \in[0, \alpha]$, and let $p, p_{1}, p_{2}, q, q_{1}, q_{2} \in(1, \infty)$ such that $\frac{1}{p}=\frac{1}{p_{1}}+\frac{1}{p_{2}}$ and $\frac{1}{q}=\frac{1}{q_{1}}+\frac{1}{q_{2}}$. Then

$$
\left\|D_{x}^{\alpha}(f g)-f D_{x}^{\alpha} g-g D_{x}^{\alpha} f\right\|_{L_{x}^{p} L_{t}^{q}} \leq c\left\|D_{x}^{\alpha_{1}} f\right\|_{L_{x}^{p_{1}} L_{t}^{q_{1}}}\left\|D_{x}^{\alpha_{2}} g\right\|_{L_{x}^{p_{2}} L_{t}^{q_{2}}} .
$$

Moreover, the following additional cases are allowed: $\left(\alpha_{1}, q_{1}\right)=(0, \infty) ;(p, q)=$ $(1,2)$; and $q=1$, provided that $\alpha_{i} \in(0, \alpha)$.

We remark that all of these results remain valid with $\tilde{D}_{x}=H D_{x}$ instead of $D_{x}$. 
Next we turn to the energy estimates satisfied by solutions to the BenjaminOno equations (1.1) and (1.2). Note that their $L^{2}$ norms are preserved by the second conservation law (1.3).

\section{Lemma 2.7.}

(a) Let $s \geq 0$ and $u \in C\left([0, T]: H^{s+2}(\mathbf{R})\right)$ be a solution to the IVP (1.1) for the Benjamin-Ono equation. Then

$$
\begin{aligned}
\sup _{t \in[0, T]}\|u(t)\|_{H^{s}} & \leq c\left\|u_{0}\right\|_{H^{s}} \exp \left(c \int_{0}^{T}\left\|\partial_{x} u(t)\right\|_{L^{\infty}} d t\right) \\
& \leq c\left\|u_{0}\right\|_{H^{s}} \exp \left(c T^{1 / 2}\left\|\partial_{x} u\right\|_{L_{T}^{2} L_{x}^{\infty}}\right) .
\end{aligned}
$$

(b) Let $s \geq 0$ and $u \in C\left([0, T]: H^{s+2}(\mathbf{R})\right)$ be a solution to the IVP (1.2) for the modified Benjamin-Ono equation. Then

$$
\begin{aligned}
& \sup _{t \in[0, T]}\|u(t)\|_{H^{s}} \leq c\left\|u_{0}\right\|_{H^{s}} \exp \left(c \int_{0}^{T}\|u(t)\|_{L^{\infty}}^{2} d t\right) \exp \left(c \int_{0}^{T}\left\|\partial_{x} u(t)\right\|_{L^{\infty}}^{2} d t\right) \\
& \operatorname{l}_{(2.20)} \leq c\left\|u_{0}\right\|_{H^{s}} \exp \left(c T^{1 / 2}\|u\|_{L_{T}^{4} L_{x}^{\infty}}^{2}\right) \exp \left(c T^{1 / 2}\left\|\partial_{x} u\right\|_{L_{T}^{4} L_{x}^{\infty}}^{2}\right) .
\end{aligned}
$$

Proof. Part (a) of the lemma is contained in [KPV1], [P]; we indicate the changes needed in the cubic nonlinearity case (b). Differentiating the equation (1.2), we have

$\partial_{t} D_{x}^{s} u+H \partial_{x}^{2} D_{x}^{s} u+\left(D_{x}^{s}\left(u^{2} \partial_{x} u\right)-u^{2} D_{x}^{s} \partial_{x} u-\left(D_{x}^{s} u^{2}\right) \partial_{x} u\right)+u^{2} \partial_{x} D_{x}^{s} u+\left(D_{x}^{s} u^{2}\right) \partial_{x} u=0$.

Multiplying by $D_{x}^{s} u$, integrating by parts, and applying Lemma 2.6 yields

$$
\begin{aligned}
\frac{d}{d t}\left\|D_{x}^{s} u(t)\right\|_{L^{2}}^{2} & \lesssim\left\|D_{x}^{s}\left(u^{2} \partial_{x} u\right)-u^{2} D_{x}^{s} \partial_{x} u-\left(D_{x}^{s} u^{2}\right) \partial_{x} u\right\|_{L_{x}^{2}}\left\|D_{x}^{s} u\right\|_{L_{x}^{2}}+ \\
& \quad+\left\|\partial_{x}\left(u^{2}\right)\right\|_{L_{x}^{\infty}}\left\|D_{x}^{s} u\right\|_{L_{x}^{2}}^{2}+\left\|\partial_{x} u\right\|_{L_{x}^{\infty}}\left\|D_{x}^{s}\left(u^{2}\right)\right\|_{L_{x}^{2}}\left\|D_{x}^{s} u\right\|_{L_{x}^{2}} \\
& \lesssim\left\|D_{x}^{s}\left(u^{2}\right)\right\|_{L_{x}^{2}}\left\|\partial_{x} u\right\|_{L_{x}^{\infty}}\left\|D_{x}^{s} u\right\|_{L_{x}^{2}}+\left\|\partial_{x}\left(u^{2}\right)\right\|_{L_{x}^{\infty}}\left\|D_{x}^{s} u\right\|_{L_{x}^{2}}^{2} \\
& \lesssim\|u\|_{L_{x}^{\infty}}\left\|\partial_{x} u\right\|_{L_{x}^{\infty}}\left\|D_{x}^{s} u\right\|_{L_{x}^{2}}^{2} .
\end{aligned}
$$

By Gronwall's inequality,

$$
\begin{aligned}
\left\|D_{x}^{s} u\right\|_{L_{T}^{\infty} L_{x}^{2}}^{2} & \leq c\left\|D_{x}^{s} u_{0}\right\|_{L_{2}}^{2} \exp \left(c \int_{0}^{T}\|u\|_{L_{x}^{\infty}}\left\|\partial_{x} u\right\|_{L_{x}^{\infty}} d t\right) \\
& \leq c\left\|D_{x}^{s} u_{0}\right\|_{L^{2}}^{2} \exp \left(c \int_{0}^{T}\left(\|u\|_{L_{x}^{\infty}}^{2}+\left\|\partial_{x} u\right\|_{L_{x}^{\infty}}^{2}\right) d t\right) .
\end{aligned}
$$

Finally, we give the key linear estimate used in the proof of Theorem 1.1 that reformulates and generalizes the one given by Koch and Tzvetkov ([KoTz]) in their demonstration of local well-posedness of BO for $s>5 / 4$. 
Proposition 2.8. Let $\alpha \in[0,1]$ and $T \in(0,1]$. Assume $w \in C\left([0, T]: H^{3}(\mathbf{R})\right)$ is a solution ${ }^{4}$ to the linear equation

$$
\partial_{t} w+H \partial_{x}^{2} w=F
$$

For any $\varepsilon>0$,

$\left\|\partial_{x} w\right\|_{L_{T}^{2} L_{x}^{\infty}} \leq c\left\|D_{x}^{1+\frac{\alpha}{4}+\varepsilon} w\right\|_{L_{T}^{\infty} L_{x}^{2}}+c\left\|D_{x}^{1-\frac{3 \alpha}{4}+\varepsilon} F\right\|_{L_{T}^{2} L_{x}^{2}}+c\|w\|_{L_{T}^{\infty} L_{x}^{2}}+c\|F\|_{L_{T}^{2} L_{x}^{2}}$.

\section{Remarks:}

(1) We take $\alpha=1 / 2$ in the proof of Theorem 1.1, which is the optimal choice of parameter in our argument. Indeed, given a linear estimate of the form

$$
\left\|\partial_{x} w\right\|_{L_{T}^{2} L_{x}^{\infty}} \lesssim\left\|D_{x}^{a} w\right\|_{L_{T}^{\infty} L_{x}^{2}}+\left\|D_{x}^{b} F\right\|_{L_{T}^{2} L_{x}^{2}}+\|w\|_{L_{T}^{\infty} L_{x}^{2}}+\|F\|_{L_{T}^{2} L_{x}^{2}}
$$

we want to apply the smoothing effect (2.5) and "absorb" as many derivatives as possible on $F$; this approach requires that $a=b+\frac{1}{2}$. Thus local well-posedness of BO holds for $s \geq a$ whenever (2.21) holds such a pair of exponents $(a, b)$. However, we have been kindly informed by L. Vega that such an interpolationtype estimate fails for any $a<\frac{9}{8}$.

(2) Koch and Tzvetkov ([KoTz]) consider instead the linearized BO equation $\partial_{t} w+H \partial_{x}^{2} w+V \partial_{x} w=F$. The strength of their estimate corresponds to the case $\alpha=1$ in the version considered here, which explains our improvement of their result by an $\frac{1}{8}$-derivative.

Proof of Proposition 2.8. Let $g=\sum_{\lambda} g_{\lambda}$ denote a Littlewood-Paley decomposition of a function $g$ (in the frequency variable dual to $x$ ), where $g_{\lambda}$ has frequency $\sim \lambda>1$ and the sum is taken over all dyadic integers. More precisely, choose $\eta \in C_{0}^{\infty}\left(\frac{1}{2}<|\xi|<2\right)$ and $\chi \in C_{0}^{\infty}(|\xi|<2)$ such that $1=$ $\sum_{k=1}^{\infty} \eta\left(2^{-k} \xi\right)+\chi(\xi)$, and for $\lambda=2^{k}$, define $g_{\lambda}=Q_{k}(g)$ where $\widehat{Q_{0} g}(\xi)=$ $\chi(\xi) \hat{g}(\xi)$ and $\widehat{Q_{k}} g(\xi)=\eta\left(2^{-k} \xi\right) \hat{g}(\xi)$ for $k \geq 1$. Recall that for $1<p<\infty$, $\|g\|_{L^{p}} \approx\left\|\left(\sum_{k}\left|Q_{k} g\right|^{2}\right)^{1 / 2}\right\|_{L^{p}}=\left\|\left(\sum_{\lambda}\left|g_{\lambda}\right|^{2}\right)^{1 / 2}\right\|_{L^{p}}$. Fix $\varepsilon>0$. For $p>1 / \varepsilon>2$, we have $\|g\|_{L^{\infty}} \lesssim\left\|J^{\varepsilon} g\right\|_{L^{p}} \approx\left\|\left(\sum_{\lambda}\left|J^{\varepsilon} g_{\lambda}\right|^{2}\right)^{1 / 2}\right\|_{L^{p}}=\left\|\sum_{\lambda}\left|J^{\varepsilon} g_{\lambda}\right|^{2}\right\|_{L^{p / 2}}^{1 / 2} \lesssim$ $\left(\sum_{\lambda}\left\|J^{\varepsilon} g_{\lambda}\right\|_{L^{p}}^{2}\right)^{1 / 2}$, so $\|g\|_{L_{T}^{2} L_{x}^{\infty}} \lesssim\left(\sum_{\lambda}\left\|J^{\varepsilon} g_{\lambda}\right\|_{L_{T}^{2} L_{x}^{p}}^{2}\right)^{1 / 2}$.

Clearly then it suffices to show that for $p>2$,

$$
\left\|\partial_{x} w_{\lambda}\right\|_{L_{T}^{2} L_{x}^{p}} \lesssim\left\|D_{x}^{1+\frac{\alpha}{4}-\frac{\alpha}{2 p}} w_{\lambda}\right\|_{L_{T}^{\infty} L_{x}^{2}}+\left\|D_{x}^{1-\frac{3 \alpha}{4}-\frac{\alpha}{2 p}} F_{\lambda}\right\|_{L_{T}^{2} L_{x}^{2}}
$$

for any frequency $\lambda=2^{k}$ with $k \geq 1$. (The case $k=0$ is easily handled using Lemma 2.1 and Hölder's inequality, yielding the last two terms in (2.21).) Fix such $\lambda \geq 2$, and observe that $\partial_{t} w_{\lambda}+H \partial_{x}^{2} w_{\lambda}=F_{\lambda}$.

Consider a partition $[0,1]=\cup I_{j}, I_{j}=\left[a_{j}, b_{j}\right]$ of the unit interval into subintervals of length $\left|I_{j}\right| \sim \lambda^{-\alpha}$, so that $T=b_{j}$ for some $j$, and note that there are

\footnotetext{
${ }^{4}$ Here we are establishing an a priori estimate for solutions $w$ having at least two derivatives in $L_{x}^{2}$, with bounds that are independent of the $H_{x}^{3}$-norms of $w$ and $F$.
} 
$O\left(\lambda^{\alpha}\right)$ of them. (For the last subinterval and the one with endpoint $T$, we require only that the length be at least $\lambda^{-\alpha}$ and at most $2 \lambda^{-\alpha}$; the other subintervals can be taken to have length $\lambda^{-\alpha}$.)

For $p>2$, set $q=4+\frac{8}{p-2}=\frac{4 p}{p-2}$. Applying Hölder's inequality, Lemma 2.1, and Jensen's inequality, we obtain (summing over indices $j$ such that $b_{j} \leq T$ )

$$
\begin{aligned}
& \left\|\partial_{x} w_{\lambda}\right\|_{L_{T}^{2} L_{x}^{p}}=\left(\sum_{j}\left\|\partial_{x} w_{\lambda}\right\|_{L_{I_{j}}^{2} L_{x}^{p}}^{2}\right)^{1 / 2} \\
& \leq \lambda^{-\alpha\left(\frac{1}{2}-\frac{1}{q}\right)}\left(\sum_{j}\left\|\partial_{x} w_{\lambda}\right\|_{L_{I_{j}}^{q} L_{x}^{p}}^{2}\right)^{1 / 2} \\
& \lesssim \lambda^{-\frac{\alpha}{2}+\frac{\alpha}{q}} \sum_{j}\left(\left\|S\left(t-a_{j}\right) \partial_{x} w_{\lambda}\left(a_{j}\right)\right\|_{L_{I_{j}}^{q} L_{x}^{p}}^{2}+\left\|\int_{a_{j}}^{t} S\left(t-t^{\prime}\right) \partial_{x} F_{\lambda}\left(t^{\prime}\right) d t^{\prime}\right\|_{L_{I_{j}}^{q} L_{x}^{p}}^{2}\right)^{1 / 2} \\
& \lesssim \lambda^{-\frac{\alpha}{2}+\frac{\alpha}{q}}\left(\sum_{j}\left\|\partial_{x} w_{\lambda}\left(a_{j}\right)\right\|_{L_{x}^{2}}^{2}+\sum_{j}\left(\int_{I_{j}}\left\|\partial_{x} F_{\lambda}\right\|_{L_{x}^{2}} d t\right)^{2}\right)^{1 / 2} \\
& \lesssim \lambda^{-\frac{\alpha}{2}+\frac{\alpha}{q}}\left(\sum_{j}\left\|\partial_{x} w_{\lambda}\right\|_{L_{T}^{\infty} L_{x}^{2}}^{2}\right)^{1 / 2}+\lambda^{-\frac{\alpha}{2}+\frac{\alpha}{q}}\left(\sum_{j} \lambda^{-2 \alpha} \lambda^{\alpha} \int_{I_{j}}\left\|\partial_{x} F_{\lambda}\right\|_{L_{x}^{2}}^{2} d t\right)^{1 / 2} \\
& \lesssim \lambda^{-\frac{\alpha}{2}+\frac{\alpha}{q}} \cdot \lambda^{\frac{\alpha}{2}}\left\|\partial_{x} w_{\lambda}\right\|_{L_{T}^{\infty} L_{x}^{2}}+\lambda^{-\alpha+\frac{\alpha}{q}}\left(\int_{0}^{T}\left\|\partial_{x} F_{\lambda}\right\|_{L_{x}^{2}}^{2} d t\right)^{1 / 2} \\
& \lesssim\left\|D_{x}^{1+\frac{\alpha}{q}} w_{\lambda}\right\|_{L_{T}^{\infty} L_{x}^{2}}+\left\|D_{x}^{1-\alpha+\frac{\alpha}{q}} F_{\lambda}\right\|_{L_{T}^{2} L_{x}^{2}} .
\end{aligned}
$$

Since $\frac{\alpha}{q}=\frac{\alpha(p-2)}{4 p}=\frac{\alpha}{4}-\frac{\alpha}{2 p}$ and $1-\alpha+\frac{\alpha}{q}=1-\frac{3 \alpha}{4}-\frac{\alpha}{2 p}$, we are done. (Note that when $p>1 / \varepsilon$ and $\alpha \leq 1$, we have $\varepsilon-\frac{\alpha}{2 p}>\frac{1}{p}-\frac{1}{2 p}=\frac{1}{2 p}>0$.)

\section{Proof of Theorem 1.1}

Fix $s>9 / 8$, and set $\varepsilon=s-9 / 8>0$. Without loss of generality, we may assume that

$$
\Lambda:=\left\|u_{0}\right\|_{L^{2}}+\left\|D_{x}^{9 / 8+\varepsilon} u_{0}\right\|_{L^{2}} \leq \delta
$$

for $\delta>0$ small enough (to be specified later). Indeed, if $u(x, t)$ is a solution to (1.1), then $u_{\lambda}(x, t)=\lambda u\left(\lambda x, \lambda^{2} t\right)$ is also a solution with initial data $\lambda u_{0}(\lambda x)$. Since $\left\|\lambda u_{0}(\lambda x)\right\|_{L^{2}}=\lambda^{\frac{1}{2}}\left\|u_{0}\right\|_{L^{2}}$ and $\left\|\lambda D^{s}\left(u_{0}(\lambda x)\right)\right\|_{L^{2}}=\lambda^{\frac{1}{2}+s}\left\|D^{s} u_{0}\right\|_{L^{2}}$, we can always reduce to the case of small initial data by rescaling. Moreover, observe that if there exists a solution $u \in C\left([0,1]: H^{s}(\mathbf{R})\right)$ whenever $\left\|u_{0}\right\|_{H^{s}} \lesssim \delta$, then for arbitrary data there exists a solution for time $T \gtrsim\left\|u_{0}\right\|_{H^{s}}^{-4}$. In the following, 
we always assume that $T \leq 1$, and we frequently apply the Cauchy-Schwarz inequality in the $t$-integrations without further comment.

In view of the energy estimate (2.19), the key point is to establish a priori control of $\left\|\partial_{x} u\right\|_{L_{T}^{2} L_{x}^{\infty}}$. Once this is done, the proofs of existence, uniqueness, and continuous dependence on initial data can be completed as in $[\mathrm{P}],[\mathrm{I}]$, [KPV1], $[\mathrm{KoTz}]$, etc.

Applying Proposition 2.8 (with $\alpha=1 / 2, w=u$, and $F=-u \partial_{x} u$ ), the energy estimate (2.19), and the $L^{2}$ conservation law, we have

$$
\begin{aligned}
\left\|\partial_{x} u\right\|_{L_{T}^{2} L_{x}^{\infty}} \lesssim & \left\|D_{x}^{9 / 8+\varepsilon} u\right\|_{L_{T}^{\infty} L_{x}^{2}}+\left\|D_{x}^{5 / 8+\varepsilon}\left(u \partial_{x} u\right)\right\|_{L_{T}^{2} L_{x}^{2}} \\
& +\|u\|_{L_{T}^{\infty} L_{x}^{2}}+\|u\|_{L_{T}^{\infty} L_{x}^{2}}\left\|\partial_{x} u\right\|_{L_{T}^{2} L_{x}^{\infty}} \\
\lesssim & \Lambda+\Lambda \exp \left(c\left\|\partial_{x} u\right\|_{L_{T}^{2} L_{x}^{\infty}}\right)+\left\|D_{x}^{5 / 8+\varepsilon}\left(u \partial_{x} u\right)\right\|_{L_{T}^{2} L_{x}^{2}}
\end{aligned}
$$

By Lemma 2.6(a) and (2.19) again,

$$
\begin{aligned}
\left\|D_{x}^{5 / 8+\varepsilon}\left(u \partial_{x} u\right)\right\|_{L_{T}^{2} L_{x}^{2}} \lesssim & \left\|u D_{x}^{5 / 8+\varepsilon} \partial_{x} u\right\|_{L_{T}^{2} L_{x}^{2}} \\
& +\left(\int_{0}^{T}\left\|D_{x}^{5 / 8+\varepsilon} u\right\|_{L_{x}^{2}}^{2}\left\|\partial_{x} u\right\|_{L_{x}^{\infty}}^{2} d t\right)^{1 / 2} \\
\lesssim & \left\|u D_{x}^{5 / 8+\varepsilon} \partial_{x} u\right\|_{L_{T}^{2} L_{x}^{2}} \\
& +\Lambda\left\|\partial_{x} u\right\|_{L_{T}^{2} L_{x}^{\infty}} \exp \left(c\left\|\partial_{x} u\right\|_{L_{T}^{2} L_{x}^{\infty}}\right) \\
\lesssim & \left\|u D_{x}^{5 / 8+\varepsilon} \partial_{x} u\right\|_{L_{T}^{2} L_{x}^{2}}+\Lambda \exp \left(c\left\|\partial_{x} u\right\|_{L_{T}^{2} L_{x}^{\infty}}\right) .
\end{aligned}
$$

Now the smoothing effect (2.5) provides a gain of half a derivative. Indeed,

$$
\begin{aligned}
\left\|u D_{x}^{5 / 8+\varepsilon} \partial_{x} u\right\|_{L_{T}^{2} L_{x}^{2}}= & \left(\sum_{j}\left\|u D_{x}^{\frac{1}{2}+\frac{1}{8}+\varepsilon} \partial_{x} u\right\|_{L^{2}([j, j+1) \times[0, T])}^{2}\right)^{1 / 2} \\
\lesssim & \left(\sum_{j}\|u\|_{L^{\infty}([j, j+1) \times[0, T])}^{2}\right)^{1 / 2} \\
& \cdot \Lambda\left(1+\Lambda+\left\|\partial_{x} u\right\|_{\left.L_{T}^{2} L_{x}^{\infty}\right)} \exp \left(c\left\|\partial_{x} u\right\|_{L_{T}^{2} L_{x}^{\infty}}\right)\right. \\
\lesssim & \left(\sum_{j}\|u\|_{L^{\infty}([j, j+1) \times[0, T])}^{2}\right)^{1 / 2} \\
& \cdot \Lambda(\Lambda+1) \exp \left(c\left\|\partial_{x} u\right\|_{\left.L_{T}^{2} L_{x}^{\infty}\right) .}\right.
\end{aligned}
$$

Using the integral equation

$$
u(t)=S(t) u_{0}+\int_{0}^{t} S\left(t-t^{\prime}\right) u \partial_{x} u\left(t^{\prime}\right) d t^{\prime}
$$


as well as Lemma 2.3 and the $L^{2}$ conservation law, we find that for fixed (small) $\eta>0$,

$$
\begin{aligned}
\left(\sum_{j}\|u\|_{L^{\infty}([j, j+1) \times[0, T])}^{2}\right)^{1 / 2} \lesssim & \Lambda+\left\|u \partial_{x} u\right\|_{L_{T}^{1} L_{x}^{2}}+\left\|D_{x}^{\frac{1}{2}+\eta}\left(u \partial_{x} u\right)\right\|_{L_{T}^{1} L_{x}^{2}} \\
\lesssim & \Lambda+\|u\|_{L_{T}^{\infty} L_{x}^{2}}\left\|\partial_{x} u\right\|_{L_{T}^{1} L_{x}^{\infty}} \\
& +\left\|D_{x}^{\frac{1}{2}+\eta}\left(u \partial_{x} u\right)\right\|_{L_{T}^{1} L_{x}^{2}} \\
\lesssim & \Lambda+\Lambda\left\|\partial_{x} u\right\|_{L_{T}^{2} L_{x}^{\infty}}+\left\|D_{x}^{\frac{1}{2}+\eta}\left(u \partial_{x} u\right)\right\|_{L_{T}^{2} L_{x}^{2}}
\end{aligned}
$$

Repeating the previous calculation (note that $\frac{1}{2}+\eta<\frac{5}{8}+\varepsilon$ ) leads to

$$
\begin{aligned}
\left\|D_{x}^{\frac{1}{2}+\eta}\left(u \partial_{x} u\right)\right\|_{L_{T}^{2} L_{x}^{2}} \lesssim & \Lambda \exp \left(c\left\|\partial_{x} u\right\|_{L_{T}^{2} L_{x}^{\infty}}\right) \\
& +\left(\sum_{j}\|u\|_{L^{\infty}([j, j+1) \times[0, T])}^{2}\right)^{1 / 2} \\
& \times \Lambda(\Lambda+1) \exp \left(c\left\|\partial_{x} u\right\|_{L_{T}^{2} L_{x}^{\infty}}\right) .
\end{aligned}
$$

Set

$$
\phi(T)=\left\|\partial_{x} u\right\|_{L_{T}^{2} L_{x}^{\infty}}+\left(\sum_{j}\|u\|_{L^{\infty}([j, j+1) \times[0, T])}^{2}\right)^{1 / 2}
$$

which is a continuous, nondecreasing function of $T$. Combining all of the preceding, we know that

$$
\phi(T) \lesssim \Lambda \exp (c \phi(T))+\Lambda(\Lambda+1) \phi(T) \exp (c \phi(T))+\Lambda+\Lambda \phi(T)
$$

so

$$
\phi(T) \leq C \Lambda+C \Lambda \exp (C \phi(T))
$$

provided that $\Lambda \leq \delta \leq 1$.

Note that

$$
\begin{aligned}
\phi(0) & =\left(\sum_{j}\left(\sup _{x \in I_{j}}|u(x, 0)|\right)^{2}\right)^{1 / 2}=\left(\sum_{j}\left\|u_{0}\right\|_{L^{\infty}\left(I_{j}\right)}^{2}\right)^{1 / 2} \\
& \lesssim\left(\left\|u_{0}\right\|_{L^{2}}^{2}+\left\|D^{s} u_{0}\right\|_{L^{2}}^{2}\right)^{1 / 2} \\
& \leq C_{0} \Lambda
\end{aligned}
$$

and take $C>C_{0}$ above.

We claim that there exists $\delta>0$ and a constant $M>0$ such that if $\Lambda \leq \delta$, then $\phi(1) \leq M$. To see this, let

$$
\Phi(y, \eta)=y-C \eta-C \eta \exp (C y) .
$$


Since $\Phi(0,0)=0$ and $\frac{\partial \Phi}{\partial y}(0,0)=1$, the implicit function theorem guarantees that for $|\eta| \leq \delta$ sufficiently small, there exists a smooth function $A(\eta)$ such that $A(0)=0$ and $\Phi(A(\eta), \eta)=0$. Clearly $A(\eta)>0$ for $\eta>0$ since $\Phi(y, \eta)<0$ for $y \leq 0$. Moreover, since $\frac{\partial \Phi}{\partial y}(0,0)=1, \Phi(\cdot, \eta)$ is increasing near $A(\eta)$ provided that $\delta$ is chosen small enough.

Assume $\Lambda \leq \delta$, and set $M=A(\Lambda)$. Then

$$
\phi(0) \leq C_{0} \Lambda<C \Lambda \leq C \Lambda+C \Lambda \exp (C A(\Lambda))=M .
$$

Suppose $\phi(T)>M$ for some $T \in(0,1)$, and let $T_{0}=\inf \{T \in(0,1): \phi(T)>M\}$. Then $T_{0}>0, \phi\left(T_{0}\right)=M$, and there exists a decreasing sequence $\left\{T_{n}\right\}$ converging to $T_{0}$ such that $\phi\left(T_{n}\right)>M$. Now $\Phi(\phi(T), \Lambda) \leq 0$ for all $T \in[0,1]$ by $(3.1)$. On the other hand, $\Phi(\cdot, \Lambda)$ is increasing near $M$, so $\Phi\left(\phi\left(T_{n}\right), \Lambda\right)>\Phi\left(\phi\left(T_{0}\right), \Lambda\right)=$ $\Phi(M, \Lambda)=\Phi(A(\Lambda), \Lambda)=0$ for $n$ sufficiently large. This is a contradiction, and we conclude that $\phi(T) \leq M$ for all $T \in(0,1)$. Hence $\phi(1) \leq M$ as claimed.

\section{Proof of Theorem 1.2}

Our main goal is to obtain a priori estimates for $\left\|\partial_{x} u\right\|_{L_{T}^{4} L_{x}^{\infty}}$ and $\|u\|_{L_{T}^{4} L_{x}^{\infty}}$ (see $(2.20)$ ), where $u$ is a solution to $\mathrm{mBO}$ and thus satisfies the integral equation

$$
u(t)=S(t) u_{0}+\int_{0}^{t} S\left(t-t^{\prime}\right) u^{2} \partial_{x} u\left(t^{\prime}\right) d t^{\prime} .
$$

(In the following, we always take $T \leq 1$.) We consider the (worse) term $\left\|\partial_{x} u\right\|_{L_{T}^{4} L_{x}^{\infty}}$ first. By Lemma 2.1 and Lemma 2.4, we have

$$
\begin{aligned}
\left\|\partial_{x} u\right\|_{L_{T}^{4} L_{x}^{\infty}} & \lesssim\left\|u_{0}\right\|_{H^{1}}+\left\|\partial_{x} \int_{0}^{t} S\left(t-t^{\prime}\right) u^{2} \partial_{x} u\left(t^{\prime}\right) d t^{\prime}\right\|_{L_{T}^{4} L_{x}^{\infty}} \\
& \lesssim\left\|u_{0}\right\|_{H^{1}}+\left\|D_{x}^{1 / 2}\left(u^{2} \partial_{x} u\right)\right\|_{L_{x}^{1} L_{T}^{2}} .
\end{aligned}
$$

Set

$$
\Lambda(T)=\sup _{p \in[1,2]}\left\|D_{x}^{1 / 2}\left(u^{2} \partial_{x} u\right)\right\|_{L_{x}^{p} L_{T}^{2}}+\left\|u^{2} \partial_{x} u\right\|_{L_{x}^{2} L_{T}^{2}} .
$$

For $p \in[1,2], p_{1} \in\left[\frac{6}{5}, 3\right], p_{2} \in[2, \infty]$ with $\frac{1}{p_{1}}+\frac{1}{6}=\frac{1}{p}$ and $\frac{1}{p_{2}}+\frac{1}{3}=\frac{1}{p_{1}}$, we apply Lemma 2.6 and Hölder's inequality to obtain

$$
\begin{aligned}
\left\|D_{x}^{\frac{1}{2}}\left(u^{2} \partial_{x} u\right)\right\|_{L_{x}^{p} L_{T}^{2}} & \leq\left\|D_{x}^{\frac{1}{2}}\left(u^{2} \partial_{x} u\right)-u^{2} D_{x}^{\frac{1}{2}} \partial_{x} u-D_{x}^{\frac{1}{2}}\left(u^{2}\right) \partial_{x} u\right\|_{L_{x}^{p} L_{T}^{2}}+ \\
& +\left\|u^{2} D_{x}^{\frac{1}{2}} \partial_{x} u\right\|_{L_{x}^{p} L_{T}^{2}}+\left\|D_{x}^{\frac{1}{2}}\left(u^{2}\right) \partial_{x} u\right\|_{L_{x}^{p} L_{T}^{2}} \\
& \lesssim\left\|u^{2} D_{x}^{\frac{1}{2}} \partial_{x} u\right\|_{L_{x}^{p} L_{T}^{2}}+\left\|D_{x}^{\frac{1}{2}} u^{2}\right\|_{L_{x}^{p_{1}} L_{T}^{6}}\left\|\partial_{x} u\right\|_{L_{x}^{6} L_{T}^{3}} \\
& \lesssim\left\|u^{2} D_{x}^{\frac{1}{2}} \partial_{x} u\right\|_{L_{x}^{p} L_{T}^{2}}+\|u\|_{L_{x}^{p_{2}} L_{T}^{\infty}}\left\|D_{x}^{\frac{1}{2}} u\right\|_{L_{x}^{3} L_{T}^{6}}\left\|\partial_{x} u\right\|_{L_{x}^{6} L_{T}^{3}} .
\end{aligned}
$$

To handle the factors in the second term, we first differentiate the integral equation for $u$ and apply the linear estimates in Lemma 2.4 (with $\theta=2 / 3$ ) to 
see that for any (positive) $\delta<1$, there exists $p=p(\delta) \in(1,2)$ such that

$$
\begin{aligned}
\left\|\partial_{x} u\right\|_{L_{x}^{6} L_{T}^{3}} \lesssim & \left\|D_{x}^{\frac{1}{3}+\frac{1}{2}+\varepsilon} u_{0}\right\|_{L^{2}}+\left\|D_{x}^{\frac{1}{3}} u_{0}\right\|_{L^{2}}+T^{\delta / 6}\left\|D_{x}^{1 / 2}\left(u^{2} \partial_{x} u\right)\right\|_{L_{x}^{p} L_{T}^{2}} \\
& +T^{1 / 2}\left\|D_{x}^{1 / 3}\left(u^{2} \partial_{x} u\right)\right\|_{L_{x}^{2} L_{T}^{2}} \\
\lesssim & \left\|u_{0}\right\|_{H^{1}}+T^{\delta / 6}\left\|D_{x}^{1 / 2}\left(u^{2} \partial_{x} u\right)\right\|_{L_{x}^{p} L_{T}^{2}}+T^{1 / 2}\left\|D_{x}^{1 / 2}\left(u^{2} \partial_{x} u\right)\right\|_{L_{x}^{2} L_{T}^{2}} \\
& +T^{1 / 2}\left\|u^{2} \partial_{x} u\right\|_{L_{x}^{2} L_{T}^{2}} \\
\lesssim & \left\|u_{0}\right\|_{H^{1}}+T^{\delta / 6} \Lambda(T) .
\end{aligned}
$$

Similarly (taking $\theta=1 / 3$ ), for any $\delta<1$, there exists $p \in[1,2]$ such that

$$
\begin{aligned}
\left\|D_{x}^{\frac{1}{2}} u\right\|_{L_{x}^{3} L_{T}^{6}} \lesssim & \left\|u_{0}\right\|_{H^{1}}+T^{\delta / 3}\left\|D_{x}^{\frac{1}{6}+\frac{\delta}{3}+\varepsilon}\left(u^{2} \partial_{x} u\right)\right\|_{L_{x}^{p} L_{T}^{2}} \\
& +T^{1 / 2}\left\|D_{x}^{\frac{1}{6}}\left(u^{2} \partial_{x} u\right)\right\|_{L_{x}^{2} L_{T}^{2}} \\
\lesssim & \left\|u_{0}\right\|_{H^{1}}+T^{\delta / 3}\left\|D_{x}^{1 / 2}\left(u^{2} \partial_{x} u\right)\right\|_{L_{x}^{p} L_{T}^{2}} \\
& +T^{1 / 2}\left\|D_{x}^{1 / 2}\left(u^{2} \partial_{x} u\right)\right\|_{L_{x}^{2} L_{T}^{2}}+T^{1 / 2}\left\|u^{2} \partial_{x} u\right\|_{L_{x}^{2} L_{T}^{2}} \\
\lesssim & \left\|u_{0}\right\|_{H^{1}}+T^{\delta / 3} \Lambda(T) .
\end{aligned}
$$

The case $\theta=0$ and the energy estimate (2.20) lead to

$$
\begin{aligned}
\|u\|_{L_{x}^{p_{2} L_{T}^{\infty}}} \lesssim & \|u\|_{L_{x}^{\infty} L_{T}^{\infty}}^{1-\frac{2}{p_{2}}}\|u\|_{L_{x}^{2} L_{T}^{\infty}}^{\frac{2}{p_{2}}} \\
\lesssim & \left(\sup _{0<t<T}\|u(t)\|_{H^{\frac{1}{2}+\varepsilon}}^{1-\frac{2}{p_{2}}}\right)\|u\|_{L_{x}^{2} L_{T}^{\infty}}^{\frac{2}{p_{2}}} \\
\lesssim & \left(\left\|u_{0}\right\|_{H^{1}}+T^{\delta / 2} \Lambda(T)\right)^{2 / p_{2}}\left\|u_{0}\right\|_{H^{1}}^{1-\frac{2}{p_{2}}} \exp \left(c T^{1 / 2}\|u\|_{L_{T}^{4} L_{x}^{\infty}}^{2}\right) \\
& \cdot \exp \left(c T^{1 / 2}\left\|\partial_{x} u\right\|_{L_{T}^{4} L_{x}^{\infty}}^{2}\right) \\
\lesssim & \left(1+\left\|u_{0}\right\|_{H^{1}}+T^{\delta / 2} \Lambda(T)\right)\left\|u_{0}\right\|_{H^{1}}^{1-\frac{2}{p_{2}}} \exp \left(c T^{1 / 2}\|u\|_{L_{T}^{4} L_{x}^{\infty}}^{2}\right) \\
& \cdot \exp \left(c T^{1 / 2}\left\|\partial_{x} u\right\|_{L_{T}^{4} L_{x}^{\infty}}^{2}\right) .
\end{aligned}
$$

We turn next to the main term in (4.2), using the smoothing effect (2.6) and the maximal function estimate (2.7) to absorb half a derivative. In detail, since 
$p \in[1,2]$ (so that $2 / p \geq 1$ ), we have

$$
\begin{aligned}
& \left\|u^{2} D_{x}^{\frac{1}{2}} \partial_{x} u\right\|_{L_{x}^{p} L_{T}^{2}}=\left(\int_{-\infty}^{\infty}\left(\int_{0}^{T}\left|u^{2} D_{x}^{\frac{1}{2}} \partial_{x} u\right|^{2} d t\right)^{p / 2} d x\right)^{1 / p} \\
& =\left(\sum_{j=-\infty}^{\infty} \int_{j}^{j+1}\left(\int_{0}^{T}\left|u^{2} D_{x}^{\frac{1}{2}} \partial_{x} u\right|^{2} d t\right)^{p / 2} d x\right)^{1 / p} \\
& \leq\left(\sum_{j=-\infty}^{\infty} \int_{j}^{j+1}\left(\sup _{0<t<T}|u|\right)^{2 p}\left(\int_{0}^{T}\left|D_{x}^{\frac{1}{2}} \partial_{x} u\right|^{2} d t\right)^{p / 2} d x\right)^{1 / p} \\
& \leq\left(\sum_{j=-\infty}^{\infty}\left(\int_{j}^{j+1}\left(\sup _{0<t<T}|u|\right)^{\frac{4 p}{2-p}} d x\right)^{\frac{2-p}{2}}\left(\int_{j}^{j+1} \int_{0}^{T}\left|D_{x}^{\frac{1}{2}} \partial_{x} u\right|^{2} d t d x\right)^{p / 2}\right)^{1 / p} \\
& \lesssim\left(\sum_{j=-\infty}^{\infty}\|u\|_{L^{\infty}([j, j+1) \times[0, T])}^{2 p}\right)^{1 / p}\left(1+\left\|u_{0}\right\|_{H^{1}}+T^{3 / 4}\left\|\partial_{x} u\right\|_{L_{T}^{4} L_{x}^{\infty}}\right)\left\|u_{0}\right\|_{H^{1}} \\
& \cdot \exp \left(c T^{1 / 2}\|u\|_{L_{T}^{4} L_{x}^{\infty}}^{2}\right) \exp \left(c T^{1 / 2}\left\|\partial_{x} u\right\|_{L_{T}^{4} L_{x}^{\infty}}^{2}\right) .
\end{aligned}
$$

Now $\left\|\left(a_{j}\right)\right\|_{\ell^{2 p}} \leq\left\|\left(a_{j}\right)\right\|_{\ell^{2}}$, so by $(2.18)$,

$$
\begin{aligned}
&\left(\sum_{j=-\infty}^{\infty}\|u\|_{L^{\infty}([j, j+1) \times[0, T])}^{2 p}\right)^{1 / p} \\
& \quad \lesssim \sum_{j=-\infty}^{\infty}\|u\|_{L^{\infty}([j, j+1) \times[0, T])}^{2} \\
& \quad \lesssim \sum_{j=-\infty}^{\infty}\left\|S(t) u_{0}\right\|_{L^{\infty}([j, j+1) \times[0, T])}^{2} \\
& \quad+\sum_{j=-\infty}^{\infty}\left\|\int_{0}^{t} S\left(t-t^{\prime}\right) u^{2} \partial_{x} u\left(t^{\prime}\right) d t^{\prime}\right\|_{L^{\infty}([j, j+1) \times[0, T])}^{2} \\
& \quad \lesssim\left(\left\|u_{0}\right\|_{H^{1}}+T^{\delta / 2}\left\|D_{x}^{1 / 2}\left(u^{2} \partial_{x} u\right)\right\|_{L_{x}^{p(\delta)} L_{T}^{2}}+T^{1 / 2}\left\|u^{2} \partial_{x} u\right\|_{L_{x}^{2} L_{T}^{2}}\right)^{2} \\
& \lesssim\left(\left\|u_{0}\right\|_{H^{1}}+T^{\delta / 2} \Lambda(T)\right)^{2}
\end{aligned}
$$

Observe that

$$
\begin{aligned}
\left\|u^{2} \partial_{x} u\right\|_{L_{x}^{2} L_{T}^{2}} \lesssim & \|u\|_{L_{T}^{\infty} L_{x}^{\infty}}\|u\|_{L_{T}^{\infty} L_{x}^{2}}\left\|\partial_{x} u\right\|_{L_{T}^{2} L_{x}^{\infty}} \\
\lesssim & T^{1 / 4}\left\|u_{0}\right\|_{L^{2}}\left\|\partial_{x} u\right\|_{L_{T}^{4} L_{x}^{\infty}}\left\|u_{0}\right\|_{H^{1}} \exp \left(c T^{1 / 2}\|u\|_{L_{T}^{4} L_{x}^{\infty}}^{2}\right) \\
& \cdot \exp \left(c T^{1 / 2}\left\|\partial_{x} u\right\|_{L_{T}^{4} L_{x}^{\infty}}^{2}\right)
\end{aligned}
$$


so we can control $\Lambda(T)$.

Finally, we need to estimate $\|u\|_{L_{T}^{4} L_{x}^{\infty}}$, which is easy. From the integral equation and the Strichartz estimate (2.3), we deduce that

$$
\begin{aligned}
&\|u\|_{L_{T}^{4} L_{x}^{\infty}} \lesssim\left\|u_{0}\right\|_{L^{2}}+\left\|u^{2} \partial_{x} u\right\|_{L_{T}^{1} L_{x}^{2}} \\
& \lesssim\left\|u_{0}\right\|_{L^{2}}+T^{3 / 4}\left\|u_{0}\right\|_{L^{2}}\left\|\partial_{x} u\right\|_{L_{T}^{4} L_{x}^{\infty}}\left\|u_{0}\right\|_{H^{1}} \exp \left(c T^{1 / 2}\|u\|_{L_{T}^{4} L_{x}^{\infty}}^{2}\right) \\
& \cdot \exp \left(c T^{1 / 2}\left\|\partial_{x} u\right\|_{L_{T}^{4} L_{x}^{\infty}}^{2}\right) .
\end{aligned}
$$

Fix $\delta<1$. We have shown that

$$
\begin{aligned}
& \Psi(T):=\max \left\{\left\|\partial_{x} u\right\|_{L_{T}^{4} L_{x}^{\infty}},\|u\|_{L_{T}^{4} L_{x}^{\infty}}, \Lambda(T)\right\} \\
& \lesssim\left\|u_{0}\right\|_{H^{1}}+\left(\left\|u_{0}\right\|_{H^{1}}+T^{\delta / 3} \Lambda(T)\right)\left(\left\|u_{0}\right\|_{H^{1}}+T^{\delta / 6} \Lambda(T)\right)\left(1+\left\|u_{0}\right\|_{H^{1}}+T^{\delta / 2} \Lambda(T)\right) \\
& \cdot\left(1+\left\|u_{0}\right\|_{H^{1}}\right) \exp \left(c T^{1 / 2}\|u\|_{L_{T}^{4} L_{x}^{\infty}}^{2}\right) \exp \left(c T^{1 / 2}\left\|\partial_{x} u\right\|_{L_{T}^{4} L_{x}^{\infty}}^{2}\right) \\
& +\left(\left\|u_{0}\right\|_{H^{1}}^{2}+T^{\delta} \Lambda(T)^{2}\right)\left(1+\left\|u_{0}\right\|_{H^{1}}+T^{3 / 4}\left\|\partial_{x} u\right\|_{L_{T}^{4} L_{x}^{\infty}}\right)\left\|u_{0}\right\|_{H^{1}} \cdot \\
& \cdot \exp \left(c T^{1 / 2}\|u\|_{L_{T}^{4} L_{x}^{\infty}}^{2}\right) \exp \left(c T^{1 / 2}\left\|\partial_{x} u\right\|_{L_{T}^{4} L_{x}^{\infty}}^{2}\right) \\
& +T^{1 / 4}\left\|u_{0}\right\|_{H^{1}}^{2}\left\|\partial_{x} u\right\|_{L_{T}^{4} L_{x}^{\infty}} \exp \left(c T^{1 / 2}\|u\|_{L_{T}^{4} L_{x}^{\infty}}^{2}\right) \exp \left(c T^{1 / 2}\left\|\partial_{x} u\right\|_{L_{T}^{4} L_{x}^{\infty}}^{2}\right) .
\end{aligned}
$$

Set

$$
\mu(T):=\max \left\{T^{\delta / 6} \Lambda(T), T^{1 / 2}\|u\|_{L_{T}^{4} L_{x}^{\infty}}^{2}, T^{1 / 2}\left\|\partial_{x} u\right\|_{L_{T}^{4} L_{x}^{\infty}}^{2}, T^{1 / 4}\left\|\partial_{x} u\right\|_{L_{T}^{4} L_{x}^{\infty}}\right\} .
$$

Note that $\mu(T)$ is continuous and $\mu(0)=0$. If $\mu(T) \leq 1$ for all $T \leq 1$, we control the $H^{s}$ norm at $T=1$. If $\mu(1)>1$, choose $T_{0} \in(0,1)$ such that $\mu\left(T_{0}\right)=1$. Then

$$
\Psi\left(T_{0}\right) \leq C+C\left\|u_{0}\right\|_{H^{1}}^{4}
$$

and one of the following inequalities holds:

$$
\begin{aligned}
1 & =T_{0}^{\delta / 6} \Lambda\left(T_{0}\right) \leq T_{0}^{\delta / 6}\left(C+C\left\|u_{0}\right\|_{H^{1}}^{4}\right) \\
1 & =T_{0}^{1 / 2}\|u\|_{L_{T_{0}}^{4} L_{x}^{\infty}}^{2} \leq T_{0}^{1 / 2}\left(C+C\left\|u_{0}\right\|_{H^{1}}^{4}\right)^{2} \\
1 & =T_{0}^{1 / 2}\left\|\partial_{x} u\right\|_{L_{T_{0}}^{4} L_{x}^{\infty}}^{2} \leq T_{0}^{1 / 2}\left(C+C\left\|u_{0}\right\|_{H^{1}}^{4}\right)^{2} \\
1 & =T_{0}^{1 / 4}\left\|\partial_{x} u\right\|_{L_{T_{0}}^{4} L_{x}^{\infty}} \leq T_{0}^{1 / 4}\left(C+C\left\|u_{0}\right\|_{H^{1}}^{4}\right)
\end{aligned}
$$

and hence $T_{0} \gtrsim\left(1+\left\|u_{0}\right\|_{H^{1}}^{4}\right)^{-6^{+}}$. At this stage, the existence, uniqueness, and continuous dependence on initial data follows from the standard compactness and Bona-Smith approximation arguments (see for example [I], [KPV1], [P]).

\section{References}

[B] T. B. Benjamin, Internal waves of permanent form in fluids of great depth, J. Fluid Mech. 29 (1967), 559-592.

[ChKi] M. Christ and A. Kiselev, Maximal functions associated to filtrations, J. Funct. Anal. 179 (2001), 409-425. 
[CKS] J. Colliander, C. E. Kenig, and G. Staffilani, Local well-posedness for dispersion generalized Benjamin-Ono equations, Preprint (2002).

[GV] J. Ginibre and G. Velo, Smoothing properties and existence of solutions for the generalized Benjamin-Ono equation, J. Differential Equations 93 (1991), 150-232.

[I] R.J. Iorio, On the Cauchy problem for the Benjamin-Ono equation, Comm. Partial Differential Equations 11 (1986), 1031-1081.

[KPV1] C.E. Kenig, G. Ponce, and L. Vega, Well-posedness of the initial value problem for the Korteweg-de Vries equation, J. Amer. Math. Soc. 4 (1991), 323-347.

[KPV2] C.E. Kenig, G. Ponce, and L. Vega, Well-posedness and scattering results for the generalized Korteweg-de Vries equation via the contraction principle, Comm. Pure Appl. Math. 46 (1993), 527-620.

[KPV3] C.E. Kenig, G. Ponce, and L. Vega, On the generalized Benjamin-Ono equation, Trans. Amer. Math. Soc. 342 (1994), 155-172.

[KoTz] H. Koch and N. Tzvetkov, On the local well-posedness of the Benjamin-Ono equation in $H^{s}(\mathbf{R})$, Int. Math. Res. Not. 26 (2003), 1449-1464.

[MR] L. Molinet and F. Ribaud, Well-posedness results for the generalized Benjamin-Ono equation with small initial data, Preprint (2003).

[MSaTz] L. Molinet, J. C. Saut, and N. Tzvetkov, Ill-posedness issues for the Benjamin-Ono and related equations, SIAM J. Math. Anal. 33 (2001), 982-988.

[O] H. Ono, Algebraic solitary waves in stratified fluids, J. Phys. Soc. Japan 39 (1975), 10821091.

[P] G. Ponce, On the global well-posedness of the Benjamin-Ono equation, Differential Integral Equations 4 (1991), 527-542.

[Sa] J.-C. Saut, Sur quelques généralisations de l'équation de Korteweg-de Vries, J. Math. Pures Appl. 58 (1979), 21-61.

[SmSo] H. F. Smith and C. D. Sogge, Global Strichartz estimates for nontrapping perturbations of the Laplacian, Comm. Partial Differential Equations 25 (2000), 2171-2183.

[St] E. M. Stein, Singular Integrals and Differentiability Properties of Functions, Princeton University Press, Princeton, 1970.

[Ta] T. Tao, Global well-posedness of the Benjamin-Ono equation in $H^{1}(\mathbf{R})$, Preprint (2003), http://arxiv.org/abs/math.AP/0307289.

[To] M. Tom, Smoothing properties of some weak solutions to the Benjamin-Ono equation, Differential Integral Equations 3 (1990), 683-694.

Department of Mathematics, The University of Chicago, Chicago, IL 60637

E-mail address: cek@math.uchicago.edu, koenig@math.uchicago.edu 